\title{
Research on the Extraction Strategy of Product Morphological Characteristics Based on Eye Tracking System
}

\author{
Yangchao XU ${ }^{\mathrm{a}, \mathrm{b}, 1}$ and Qun WU ${ }^{\mathrm{c}, \mathrm{d}}$ \\ ${ }^{a}$ Art and Design School, Zhejiang Sci-Tech University, Hangzhou (PR China) \\ ${ }^{\mathrm{b}}$ Ouhai Research Institute of Zhejiang Sci-Tech University, Wenzhou (PR China) \\ ${ }^{\mathrm{c}}$ Collaborative Innovation Center of Culture, Creative Design and Manufacturing \\ Industry of China Academy of Art, Hangzhou (PR China) \\ ${ }^{\mathrm{d}}$ Zhejiang Provincial Key Laboratory of Integration of Health Smart Kitchen \\ System, Hangzhou (PR China)
}

\begin{abstract}
This article mainly takes a textile equipment product as an example to study the strategy of extracting the relevant information of its product morphology characteristics. The research mainly takes the eye tracking system as the main technical means, combined with the methods of perceptual survey, extracts the user's visual perception information caused by the morphological characteristics of the product. This strategy eliminates the influence of other appearance styling factors, and conducts experiments on the premise that only product shape information is retained to ensure the correlation between the product shape and the user's visual experience; through the use of eye tracking technology for quantitative analysis and perceptual evaluation research Combining qualitative analysis, and on the basis of saving costs and improving efficiency, it obtains more objective and effective visual feedback information from users on the appearance of the product, provides a more stable and reliable idea for related industrial equipment manufacturers to break through the cost and space restrictions in the product design process to obtain user feedback information, thereby improving the fit between the product appearance and user needs.
\end{abstract}

Keywords. Morphological characteristics; Control variable; Eye tracking system; Perception evaluation survey; Product style

\section{Introduction}

\subsection{Research background and current situation}

For enterprises in the field of industrial equipment manufacturing, the lack of innovation and brand style characteristics in appearance design will lead to product homogeneity, which will seriously affect the product's market competitiveness. At the same time, the lack of consideration of the actual needs of users in the appearance design will bring adverse effects and hidden dangers to the use of the product. In actual development, this type of enterprises will face to multiple constraints. For example, it is

\footnotetext{
${ }^{1}$ Corresponding Author. Qun Wu, 5 Second Avenue, Xiasha Higher Education Zone, Hangzhou, China; E-mail:wuq@zstu.edu.cn
} 
difficult to systematically collect experience information due to the confidentiality of the use process; The variety and quantity of products also create a huge cost barrier to the research needed for style improvement. These problems cause companies to lack research on user experience information, which makes them have to rely more on personal experience and subjective feelings when designing appearances, lead to reduces the degree of fit with users' actual feelings; however, it is difficult to modify and adjust the plan on a large scale due to material and technical limitations during the realization of the plan. It is precisely that there is an urgent need for accurate and efficient acquisition of research information. Therefore, exploring new ideas to solve the problems is of great significance to helping enterprises to get rid of predicaments and achieve excellent style and appearance designs.

\subsection{Overview of related theories}

\subsubsection{Eye tracking technology}

Eye tracking technology is a technical method that measures the direction of the line of sight based on the characteristics of eye movement to determine the position of the eye point of the human eye. It is also a method of recording true, natural and objective user behavior [1]. The gaze tracking and analysis system built by this technology is the eye tracking system. The eye tracking experiment is the core of the system, use conventional quantitative methods to analyze user cognitive decision-making by obtaining eye movement data. Compared with traditional methods, the eye tracking system is faster and more efficient, more objective and accurate, and can be used to explore the laws of human cognition, which is conducive to understanding and mastering the essence of human learning [2]. The important basis for the system to obtain users' visual experience are three visual behavior indicators: Fixation, Saccade, Glance. The method of dividing the observed image area of interest (AOI) for analysis is the key method of the system, which is the focus of this research.

\subsubsection{Product morphological characteristics}

In industrial design, product modeling includes a variety of elements such as form, color, material, and texture. Product form is the most basic one, which determines the basis and way of product's performance in the material field. The realization of other elements must be based on it and subject to its constraints. Product characteristics refer to the characteristics that can express the product from a certain aspect and are different from other similar products, and all the characteristics sets constitute the features of the product itself [3], then become the basis for style formation. As far as products are concerned, the formation of style includes not only the physical cognition and discrimination of users brought by the commonality of modeling characteristics, but also the psychological and emotional impact of characteristics on users [4]. From the perspective of product design, the process of it can be regarded as a characteristic evolution process [5]. From the perspective of product form, the characteristics of form constitute the basic unit of the overall appearance structure of a product. It not only has geometric information at the physical level, but also contains the emotional cognition brought to users at the psychological level, including visual feeling, imagery cognition, etc., called morphological characteristics. Its shape characteristics and spatial arrangement relationship reflect the specific style of this product, which can be said to be the basic unit forming the style situation [6]. Therefore, the research on morphological 
characteristics is valuable to the improvement of product appearance and style. There have been researches on product characteristics that have used eye tracking technology, such as Lisha Ma et al.'s analyzed the modeling characteristics of automobiles [7], Ruiting Yang's research on UAV modeling characteristics [8], etc. The type of research has defects such as not restricting styling elements and less analysis material, so their validity needs to be improved.

\section{Subject research work}

\subsection{Propose the extraction strategy and the research framework}

To solve the problems, this research uses textile equipment products as a carrier to propose a strategy for extracting information related to product morphological characteristics. The purpose is to clarify the user's focus and style tendency of the product's appearance through the use of eye tracking technology, to provide a reliable basis for subsequent product design optimization. And on the basis of saving costs and improving efficiency, it helps designers in manufacturing field to quickly collect user visual perception information, help to solve related design dilemmas. Steps includes: the screening and preprocessing of typical products; the product morphological characteristics extraction experiment based on the eye tracking system; the supplement of the perception evaluation survey, as Figure 1.

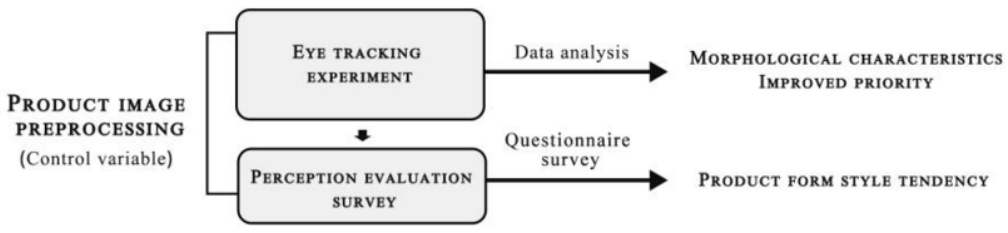

Figure 1. Extraction strategy framework

The core of the extraction strategy is to obtain and analyze the user's visual perception information brought by the product form characteristics. The innovation is to use eye tracking system to transform the traditional evaluation method of design schemes with subjective perception evaluation as the core to quantitative analysis and evaluation based on objective data as the core, improving the screening efficiency and accuracy of programs. At the same time, by preprocessing the experimental materials, it eliminates the other modeling factors, it uses control variables to improve the correlation between the user feelings reflected by the eye movement data and the product form.

\subsection{Typical product screening and material handling}

It is necessary to screen the design objects in a targeted manner to ensure that the design strategy proposed by the research has strong applicability. Based on the precollected products, after dividing by structural differences and considering the actual production situation, we selected the textile cool feeling tester in the desktop equipment as a typical product for strategic research finally, as Figure 2. 


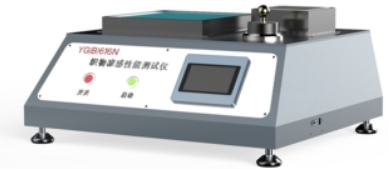

Figure 2. Schematic of the original product rendering

Figure 3. Example of wireframe rendering product morphological characteristics is as strong as possible after the following eye tracking experiment, it is necessary to exclude the influence of factors such as color, material, texture, lighting effect, and shadow. After the feasibility analysis of related technologies, it was decided to wireframe the typical product: import product model into KeyShot 9 for re-rendering; remove the shadow effect, set the global illumination to avoid the brightness difference of the light from different angles; give each part a cartoon type material to achieve a wireframe effect, make the thickness and brightness of the border consistent. The surface of the product is set to pure white and diffuse reflection effect, as Figure 3.

\subsection{Eye tracking experiment and data analysis}

\subsubsection{Experiment preparation and process}

Through plane image observation, the eye movement data was collected for the observation activities of the tested products, and analyze the participants' visual feelings, clarify the degree of difference in the feelings caused by morphological characteristics. The participants in this experiment are 10 men and women each, with an average age of about 24 years old. They were required to have normal naked vision, no color blindness and color weakness, and no neurological diseases, and were required to have no professional understanding of industrial equipment in the manufacturing sector.

- Experimental materials and equipment

The experimental materials come from slides which made from product images of various perspectives (as Figure 4), and the images are displayed in a clockwise order (vertical view is the last). When playing a slideshow, the time of each view is $10 \mathrm{~s}$, and there is a $5 \mathrm{~s}$ black screen before and after the slide and between every two views to help eliminate the influence of the previous picture. Each page is set to switch quickly at a speed of $0.1 \mathrm{~s}$. Sight capture device is Tobii Glass 2 eye tracker.

- Experiment process

Complete the relevant preparatory activities before starting the eye tracking experiment. The scene is as Figure 4. The detailed operation process is as follows:

1) Complete the settings of the equipment and software, fill in the information of the participants;

2) Read the experiment instruction and make the participants fully understand, emphasizing the correct sitting posture during the experiment. After that, wear the eye tracker for them and make adjustments to ensure that they are comfortable to wear;

3) Sight calibration: Judge the effect of gaze by looking at the center of the circle.

After gaze correction, the experiment started. Tobii Glasses Controller was used to play a slide. After the playback, the participants fill out the survey questionnaire for perception evaluation. 


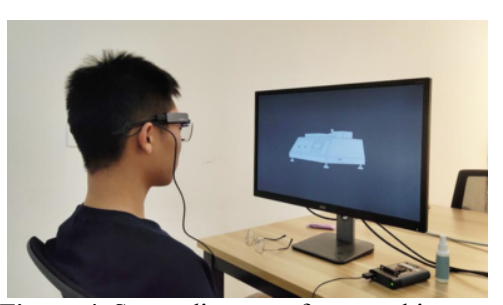

Figure 4. Scene diagram of eye tracking experiment

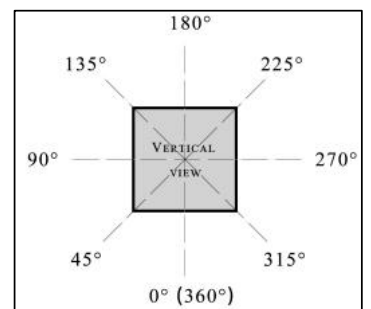

Figure 5. Experimental observation angle

\subsubsection{Analysis of results}

This experiment mainly uses the method of dividing the region of interest to analyze the data through eye movement indicators, and uses three kinds of eye movement indicators for statistical analysis, including: Number of fixations, Total duration of fixations, Number of visits. The reason for using the above three indicators is that the analysis results through them are more intuitive and typical. Indeed, gaze (fixation) duration has been used as the preferred dependent variable in much psycholinguistic research using eye-movement measures [9].

By dividing the region of interest, the relevant eye movement indicators can be quantitatively analyzed. Summarize the eye movement data according to the eye movement indicators, as Table1.

Table 1. The main indicators of typical product eye movement experimental data

\begin{tabular}{|c|c|c|c|c|}
\hline & & $\begin{array}{l}\text { Number of } \\
\text { fixation }\end{array}$ & $\begin{array}{l}\text { Total duration of } \\
\text { fixations }(\mathrm{ms})\end{array}$ & $\begin{array}{c}\text { Number } \\
\text { of visits }\end{array}$ \\
\hline \multirow{5}{*}{$\begin{array}{c}0^{\circ} \\
\left(360^{\circ}\right)\end{array}$} & Touch panel & 79 & 34301 & 61 \\
\hline & Base & 28 & 8877 & 23 \\
\hline & Sensor & 46 & 23546 & 40 \\
\hline & Switch button & 59 & 26826 & 48 \\
\hline & $\begin{array}{c}\text { Sample } \\
\text { placement platform }\end{array}$ & 31 & 7677 & 30 \\
\hline \multirow{5}{*}{$45^{\circ}$} & Touch panel & 36 & 15333 & 30 \\
\hline & Base & $\begin{array}{l}36 \\
35\end{array}$ & $\begin{array}{l}15333 \\
11513\end{array}$ & 24 \\
\hline & Sensor & 49 & 23049 & $\begin{array}{l}24 \\
35\end{array}$ \\
\hline & Switch button & 45 & 21265 & 32 \\
\hline & $\begin{array}{c}\text { Sample } \\
\text { placement platform }\end{array}$ & 52 & 16411 & 37 \\
\hline \multirow{2}{*}{$90^{\circ}$} & Base & 54 & 26404 & 38 \\
\hline & $\begin{array}{c}\text { Sample } \\
\text { placement platform }\end{array}$ & 108 & 57227 & 56 \\
\hline \multirow{4}{*}{$135^{\circ}$} & Base & 26 & 10375 & 23 \\
\hline & Heater & 8 & 2399 & 4 \\
\hline & Interface 1 & 46 & 40378 & 32 \\
\hline & $\begin{array}{c}\text { Sample } \\
\text { placement platform }\end{array}$ & 45 & 19187 & 40 \\
\hline \multirow{4}{*}{$180^{\circ}$} & Base & & 15552 & \\
\hline & Heater & 25 & 8715 & 25 \\
\hline & Interface 1 & 97 & 71639 & 56 \\
\hline & $\begin{array}{c}\text { Sample } \\
\text { ate }\end{array}$ & 17 & 4978 & 16 \\
\hline \multirow{6}{*}{$225^{\circ}$} & Base & 63 & 30185 & 46 \\
\hline & Sensor & 13 & 3700 & 12 \\
\hline & Heater & 34 & 13952 & 28 \\
\hline & Interface2 & 20 & 13574 & 17 \\
\hline & Interface1 & 70 & 34540 & 51 \\
\hline & Sample & 11 & 3558 & 10 \\
\hline
\end{tabular}




\begin{tabular}{|c|c|c|c|c|}
\hline & placement platform & & & \\
\hline \multirow{4}{*}{$270^{\circ}$} & Base & 87 & 40336 & 62 \\
\hline & Sensor & $\begin{array}{l}81 \\
43\end{array}$ & $\begin{array}{l}40350 \\
27066\end{array}$ & 33 \\
\hline & Interface2 & 49 & 31281 & 38 \\
\hline & Sample & 15 & 7357 & 14 \\
\hline \multirow{8}{*}{$315^{\circ}$} & $\begin{array}{r}\text { placement plattorm } \\
\text { Touch panel }\end{array}$ & & & \\
\hline & Base & 67 & 35917 & 61 \\
\hline & Base & 52 & 16812 & 30 \\
\hline & Sensor & 48 & 20748 & 41 \\
\hline & $\begin{array}{l}\text { Heater } \\
\text { Interface? }\end{array}$ & 6 & 1679 & 6 \\
\hline & $\begin{array}{c}\text { Interface } 2 \\
\text { S }\end{array}$ & 12 & 4358 & 11 \\
\hline & Switch button & 27 & 18071 & 22 \\
\hline & $\begin{array}{c}\text { Sample } \\
\text { placement platform }\end{array}$ & 21 & 10075 & 17 \\
\hline \multirow{6}{*}{ view ${ }^{\text {Vertical }}$} & Touch panel & 30 & 10656 & 27 \\
\hline & Sensor & 42 & 19371 & 41 \\
\hline & Heater & 75 & 31680 & 52 \\
\hline & Switch button & 14 & 6337 & 12 \\
\hline & Interfacel & 6 & 1999 & 6 \\
\hline & $\begin{array}{c}\text { Sample } \\
\text { placement platform }\end{array}$ & 74 & 22748 & 54 \\
\hline
\end{tabular}

In order to more intuitively explain the difference in the influence of the morphological characteristics, this study uses the concept of eye-tracking appearance attractiveness index to evaluate [10]. In this method, the product appearance attractiveness is regarded as a normal distribution, due to the controlled variable processing of the experimental observation materials in the research, the appearance index will be strictly limited to the influence of morphological characteristics. According to the division of AOI, it can be considered that this kind of influence can basically be regarded as the influence of the morphological characteristics in the AOI in the experiment. After that, by calculating the average value $(\mathrm{X})$ of the eye movement data in each AOI of a view, and then we can obtain the standard deviation $(\sigma)$ of the eye movement data, and therefore the standard score $(Z)$ can be obtained, which represents its position in the normal distribution graph. The appearance attractiveness index is the average of the standard scores of the eye tracking data of the region of interest in each view. By sorting the average number, the gradient of the product appearance attractiveness index in the AOI of each view can be obtained, which is defined as "Total ranking".

Table 2. Details and ranking of the AOI Appearance Attractiveness Index of each view of the product

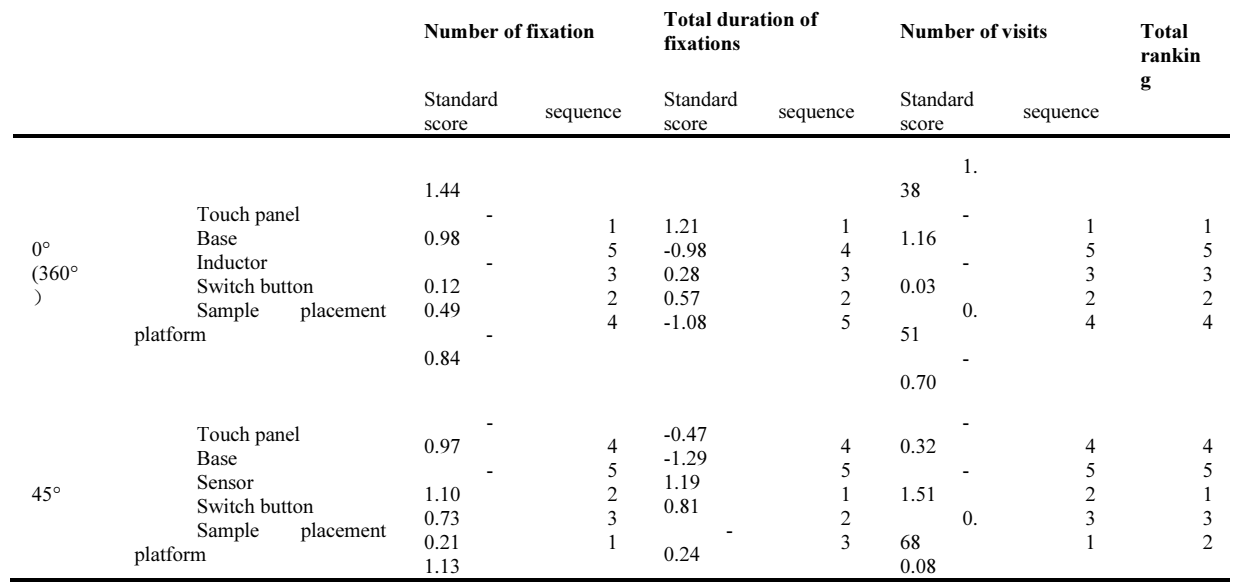




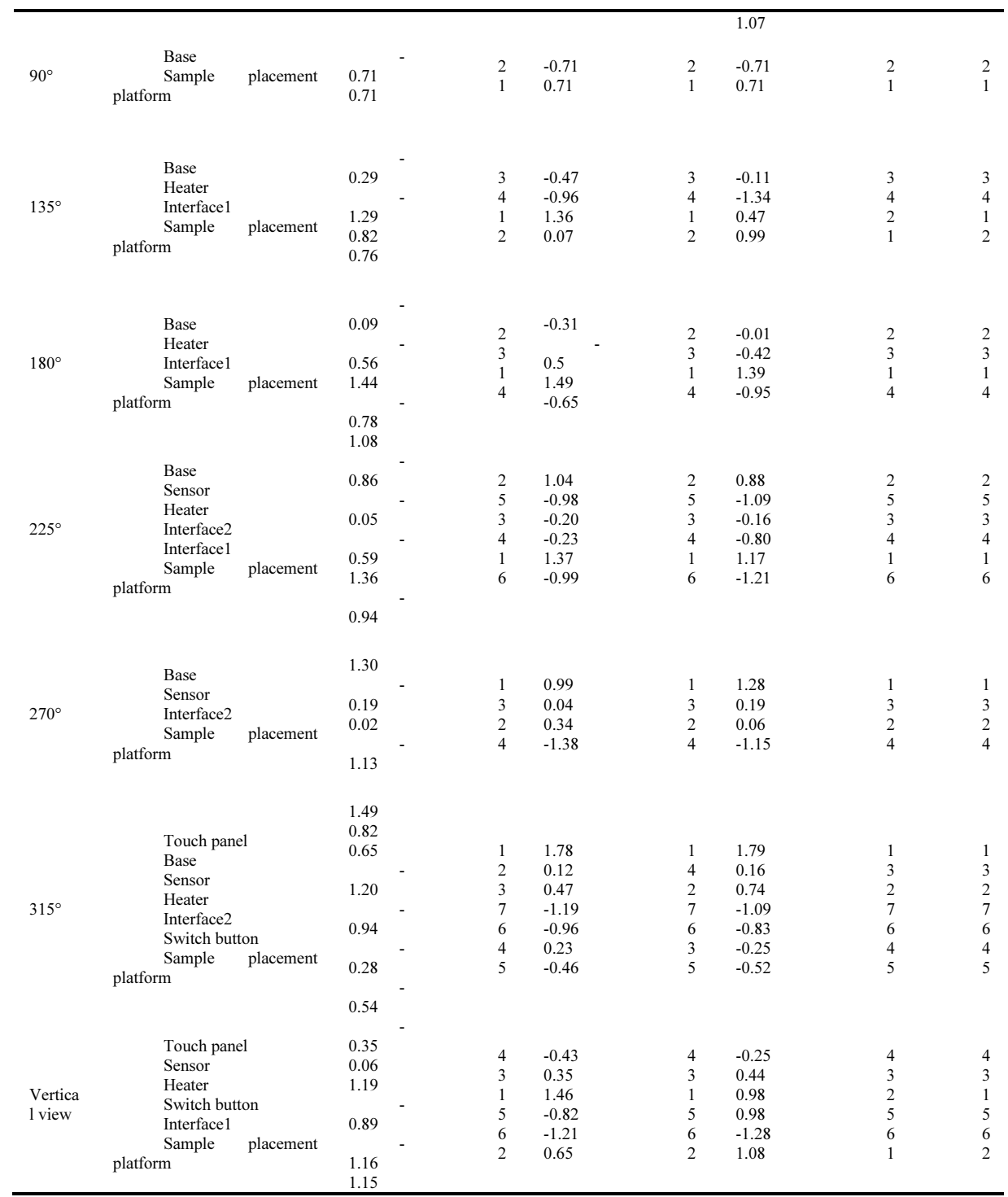

For the same view, the higher the total ranking of the eye movement indicators corresponding to the AOI, the higher the appearance attractiveness index of the AOI in this view, that is, the higher the appearance attractiveness index of the product morphological characteristics in the area. For a certain eye movement indicator in a view, the higher the ranking of "Number of fixations" and "Total duration of fixations", the longer the time that the product morphology characteristics in the AOI attracts the participant's attention; the higher the ranking of "Number of visits", the more often the participants have shifted their sights to the AOI.

Analysis of the table, after sorting the AOI appearance attractiveness index in each view, we can get the ranking of user's visual attention to the product morphological characteristics represented by the AOI in each view, and then get the priority of improved design for morphological characteristics. The priority reflects participants' different 
visual feelings of the characteristics from different perspectives. Due to the differences in the number and types of product characteristics observed in each perspective, the source of the feelings includes not only the different effects produced by same characteristic from different perspectives, but also the different observation effects produced by different types of characteristics. Considering the differences, it's necessary to combine the ranking results of "whole" and "partial" for analysis when judging the priority of each AOI when improving the design.

From the perspective of partial subdivision, in the front view of typical products, the top three are "Touch panel", "Switch button", "Sensor"; at $45^{\circ}$ angle of view, the top three are "Sensor", "Sample" "placement platform", "Switch button"; at $90^{\circ}$, the number one is "Sample placement platform", the top two are "Interface1", "Sample placement platform"; at $180^{\circ}$, the top two are "Interface1", "Base"; at $225^{\circ}$, the top three are "Interface1", "Base", "Heater"; at $270^{\circ}$, the top two are "Base" and "Interface2"; at $315^{\circ}$, the top four are "Touch panel", "Sensor", "Base", "Switch button"; in the vertical view, the top three are "Heater", "Sample placement platform", "Sensor".

From all perspectives as a whole, it is necessary to use the weighted average method to calculate the appearance attractiveness index of each view AOI. The calculation method is as $\operatorname{Eg}(1)$ :

$$
\left\{\begin{array}{l}
\bar{T}=\frac{T_{1} f_{1}+T_{2} f_{2}+T_{3} f_{3}+\cdots \cdots+T_{k} f_{k}}{n} \\
n=f_{1}+f_{2}+f_{3}+\cdots \cdots+f_{k} \quad(k \in N+)
\end{array}\right.
$$

" $T$ " is the number of total rankings; " $T$ " is the weighted average of the number of total rankings; " $f$ " is the times that each total ranking appears; " $n$ " is the total number of the AOI appears in all views; " $k$ " is the number that total ranking sorted by types.

According to the number of times the total ranking appears in all views, we have performed a new overall ranking from the ranking digits of the appearance attractiveness index corresponding to the various types of products AOI (It is the weighted average of the number of total rankings of a certain type AOI in each view), as Table 3.

Table 3. Overall ranking of various AOI appearance attractiveness indexes of typical product

\begin{tabular}{|c|c|c|c|c|c|c|c|c|}
\hline $\begin{array}{l}\text { Total } \\
\text { ranking }\end{array}$ & $\begin{array}{l}\text { Touch } \\
\text { panel }\end{array}$ & Base & Sensor & Heater & Interface 2 & $\begin{array}{l}\text { Switch } \\
\text { button }\end{array}$ & Interface1 & $\begin{array}{c}\text { Sample } \\
\text { placement } \\
\text { platform }\end{array}$ \\
\hline 1 & 2 & 1 & 1 & 1 & & & 3 & \\
\hline 2 & & 3 & 1 & & 1 & 1 & & 3 \\
\hline 3 & & 2 & 3 & 2 & & 1 & & 2 \\
\hline 4 & 2 & & & 1 & 1 & 1 & & 3 \\
\hline 5 & & 2 & 1 & & & 1 & & 1 \\
\hline 6 & & & & & 1 & & 1 & 1 \\
\hline 7 & & & & 1 & & & & \\
\hline $\begin{array}{c}\text { Total } \\
\text { occurrences }\end{array}$ & 4 & 8 & 6 & 7 & 3 & 4 & 4 & 9 \\
\hline $\begin{array}{l}\text { Weighted } \\
\text { average of total } \\
\text { rankings }\end{array}$ & 2.5 & 2.5 & 2.83 & 2.26 & 4 & 3.5 & 2.25 & 3.44 \\
\hline
\end{tabular}

From the above table, after summarizing the product attractiveness index of various AOI as a whole, the priority of the product morphological characteristics represented by each AOI when improving the design is: "Interface1" - "Heater" - "Touch panel" / "Base" - "Sensor" - "Sample placement platform" - "Switch button" - "Interface2" (From high 
to low). Considering the actual use of the product, the back side often does not receive visual attention most of the time, so the subsequent improvement design should give priority to the characteristics on the other sides. In the above rankings, the priority of AOI on the back is transferred to the end, the final ranking is: "Heater" - "Touch panel" / "Base" - "Sensor" - "Sample placement platform" - "Switch button" - "Interface1" "Interface2".

\subsection{Perception evaluation survey}

The purpose of the perceptual evaluation survey is to clarify the specific tendency of the attraction effect that the survey is carried out on the basis of the participants' visual attraction ranking of eye movement experiment obtained by various types of AOI, so that the follow-up product form improvement can be more targeted and directional, and it is an effective supplement to the analysis results of the eye movement experiment data.

The survey uses the scoring evaluation method and the combination of closed and open questions for investigation. The content was divided into immediate investigation and analytical investigation. The former ensures that the real-time feelings of participants in the experiment are fully recorded; the latter collects feedback information when the participants have a full experience of the images and a certain degree of understanding of related product information. The survey mainly Includes two aspects: the participants' intuitive image recognition of the product morphological characteristics and the degree of understanding of functions through the morphological characteristics. The former mainly allows the participants to choose the product image and supplement first impression of the product form; the latter mainly investigates the participants' understanding of morphological characteristics function after observing the experiment (as Table 4), and investigates the expectations for style improvement (as Table 5), at the same time according to the expectations of the choice to compare scores, as Table 6.

Table 4. The AOI that the participants have doubts about the function in the experiment

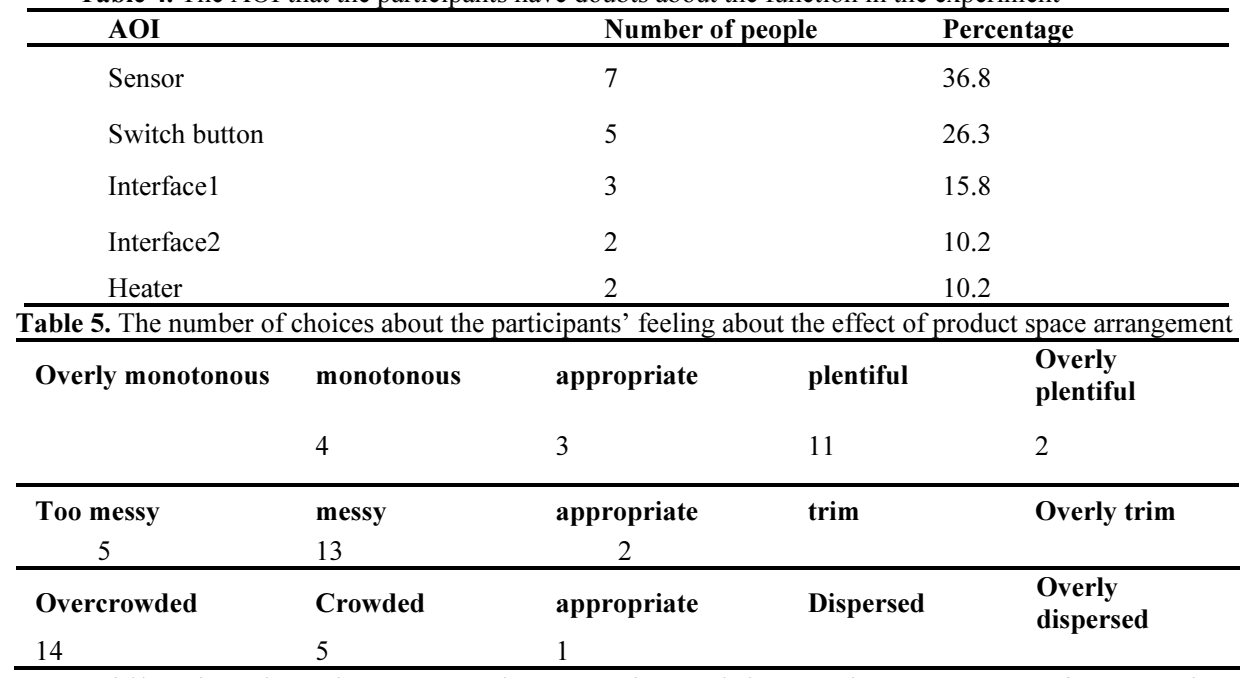

While choosing the expectations, each participant chooses up to four product imageries, as Table 6: 
Table 6. Participants' expectations of product form and style improvement.

\begin{tabular}{lcccc}
\hline & $\begin{array}{c}\text { A little expectation } \\
\text { Score (1) }\end{array}$ & $\begin{array}{c}\text { Medium expectations } \\
\text { Score (2) }\end{array}$ & $\begin{array}{c}\text { High expectations } \\
\text { Score (3) }\end{array}$ & Total score \\
\hline Plentiful & 2 & 0 & 0 & 2 \\
Concise & 2 & 7 & 6 & 34 \\
Lightness & 3 & 6 & 2 & 21 \\
Heavy & 2 & 0 & 0 & 2 \\
Sharp & 0 & 0 & 0 & 0 \\
Mellowed & 6 & 5 & 7 & 37 \\
Plain & 3 & 0 & 0 & 3 \\
Fashion & 3 & 6 & 12 & 51 \\
\hline
\end{tabular}

For this product, the number of participants who felt that it should be adjusted to the "fashion" style was the largest, followed by "roundness" and "concise", followed by "lightness"; Let the participants check the morphological characteristics that have the greatest impact on the overall visual effect, and the top three choices are: "Switch button", "Sensor", "Interface1". This result is basically consistent with the ranking obtained after processing the actual eye movement data, which further illustrates that the user situation reflected by the data is highly authentic. The most controversial combination method of product AOI is "Sensor" - "Touch panel" - "Switch button". This means the spatial relationship between them needs to be adjusted first in subsequent improvements. Since most of these components are not placed on the same plane, adjusting their spatial relationship will have a greater impact on the overall structure of the product. Therefore, in subsequent design activities, attention should be paid to adjusting them at the height of the overall planning of the product.

\section{Summary and prospects}

This study explored the way of user information collection in design activities through a combination of eye tracking systems and qualitative surveys, provides new ideas for enterprises in this field to solve related design problems. In other way, when using this extraction strategy to research multiple typical products, the advantages can be further exerted. It can achieve lower cost and higher efficiency in guiding the subsequent optimization of product morphological characteristics and stylized improvement, helping enterprises in this field to enhance the market competitiveness of products.

\section{Acknowledgment}

This work is supported by Zhejiang Provincial Key Laboratory of Integration of Smart kitchen System (Grant No: 19080049-N)

\section{References}

[1] Junjie Zhang, Guangmin Sun, Kun Zheng. Review of gaze tracking and its application in intelligent education, Journal of Computer Applications, 40 (2020), 11, 3346-3356.

[2] Xin Sun, Qinglong Zhan. Visual Analysis of the Literature on Eye Tracking Technology from the Perspective of High Frequency Terminology, China Terminology. 23 (2021), 1, 71-80. 
[3] Zuyao Zhang, Yuan Zhu. Research on product design based on morphological characteristics analysis. Modern Decoration(theory), 9 (2013), 98.

[4] Kai Wang. Exploration on Cognition of Product Formal based on Semiology and Aesthetic, Zhejiang University 2004.

[5] Shouqian Sun, Weien Bao, Yunhe Pan. Component Feature Model For Product Layout Design. Journal of Computer-Aided Design and Computer Graphics. 1 (1990), 29-33.

[6] Chan C S. Can style be measured?. Design Studies, 21 (2000), 3, 277-291.

[7] Lisha Ma, Jian Lv, Junjun Shan, Weijie Pan. Design Method of Automobile Modeling Feature Line Based on Eye Movement Tracking, Packaging Engineering, 40 (2019), 4, 234-241.

[8] Ruiting Yang. Research on Recognition of Form Features of UAV based on Eye tracking. Design, 32 (2019), 2, 141-143.

[9] Binder, Katherine,S., Pollatsek, Alexander, \& Rayner, K. Extraction of information to the left of the fixated word in reading. Journal of Experimental Psychology: Human Perception and Performance, 25 (1999), 4, 1162-1172.

[10] Qi Huang. Research on the Technology of Computer-aided Conceptual Design based on Product Style Recognition Model, Zhejiang University 2005. 